Bulletin of the Section of Logic

Volume 46:1/2 (2017), pp. 93-109

http://dx.doi.org/10.18778/0138-0680.46.1.2.08

Nobu-Yuki Suzuki

\title{
SOME WEAK VARIANTS OF THE EXISTENCE AND DISJUNCTION PROPERTIES IN INTERMEDIATE PREDICATE LOGICS*
}

Dedicated to Professor Grzegorz Malinowski

\begin{abstract}
We discuss relationships among the existence property, the disjunction property, and their weak variants in the setting of intermediate predicate logics. We deal with the weak and sentential existence properties, and the Z-normality, which is a weak variant of the disjunction property. These weak variants were presented in the author's previous paper [16]. In the present paper, the Kripke sheaf semantics is used.
\end{abstract}

Keywords: intermediate predicate logics, existence property, disjunction property

\section{Introduction}

We discuss relationships among the existence property (EP), disjunction property (DP), and their weak variants in the setting of intermediate predicate logics by making use of the Kripke sheaf semantics. We deal with the weak and sentential existence properties, and the $Z$-normality, which is a weak variant of the disjunction property. These weak variants were treated in the author's previous paper [16] in which intermediate predicate logics

*Supported in part by Grant-in-Aid for Scientific Research (C) No. 24540120, Japan Society for the Promotion of Science. 
having EP but lacking DP were constructed. This result revealed that EP and DP are independent in intermediate predicate logics ${ }^{1}$.

An intermediate predicate logic $\mathbf{L}$ is said to have $\mathrm{EP}$, if the following condition holds: for every $\exists x A(x), \mathbf{L} \vdash \exists x A(x)$ implies that there is an individual variable $v$ such that $\mathbf{L} \vdash A(v)$. (For the precise definition of EP, see Definition 1.2.) An intermediate predicate logic $\mathbf{L}$ is said to have DP, if the following condition holds: for every $A$ and every $B, \mathbf{L} \vdash A \vee B$ implies that $\mathbf{L} \vdash A$ or $\mathbf{L} \vdash B$. In [16], we constructed intermediate predicate logics having EP but laking DP by making use of two variants of EP. One variant is an extremely strong EP, which can be meaningful in super-intuitionistic predicate logics; namely, for every $\exists x A(x), \mathbf{L} \vdash \exists x A(x)$ implies that there exists a fresh individual variable $v$ such that $\mathbf{L} \vdash A(v)$. This property is so extreme that none of the intermediate predicate logics has it. However, if we restrict $\exists x A(x)$ to a sentence, we obtain one weak variant of $\mathrm{EP}$, which we call here the sentential existence property ( $\mathrm{sEP}$ ).

Another one is a weak EP; an intermediate predicate $\operatorname{logic} \mathbf{L}$ is said to have the weak existence property (wEP), if for every $\exists x A(x), \mathbf{L} \vdash \exists x A(x)$ implies that there exist finitely many terms $t_{1}, \ldots, t_{n}$ in the vocabulary of $\exists x A(x)$, the disjunction $A\left(t_{1}\right) \vee \cdots \vee A\left(t_{n}\right)$ is provable in $\mathbf{L}$. (For the precise definition of $\mathrm{wEP}$ in the setting of intermediate predicate logics, see Definition 1.4.) In [16], we proved EP of some logics by showing wEP and $\mathrm{DP}^{2}$.

Moreover, we introduced a weak variant of DP called $Z$-normality (For the definition of $Z$-normality, see Definition 1.6). The $Z$-normality is a natural property for semantically reasonable logics ( $c f$. Proposition 2.4), and is important in the consideration of the relation between EP and DP. Namely, every $Z$-normal logic with EP always has DP.

In this paper, we show relationships among EP, wEP, sEP, $Z$-normality, and their combinations in intermediate predicate logics.

The essential ideas come from Nakamura [9]'s and Minari [8]'s constructions. They used the Kripke frame semantics, but we use the Kripke sheaf semantics, since Kripke sheaves enable us to handle our modifications of models easily.

\footnotetext{
${ }^{1}$ This is a negative solution to one of problems of Umezawa, Minari, and Ono ([18, 8, 11]): Does EP imply DP?

${ }^{2}$ This procedure is based on the idea of in Prawitz [12], Komori [7], and Minari [8]; they proved EP of intuitionistic predicate logic and some intermediate predicate logics in this way.
} 
$\S 1$ contains some preliminaries to make this paper rather self-contained. The main theorem is presented at the end of $\S 1$. $\S 2$ provides semantical preliminaries. Readers having some knowledge of the Kripke sheaf semantics, especially one of the author's [13], [14, §1], [15], or Gabbay-ShehtmanSkvortsov [3, Chapter 3], may skip §2.1. Semantical tools for proving the main result are given in $\S 2.2$. The proof of main result is presented in $\S 3$. The last $\S 4$ is devoted to concluding remarks.

\section{Preliminaries and the main Theorem}

We fix a pure first-order language $\mathcal{L}$, which consists of logical connectives $\vee$ (disjunction), $\wedge$ (conjunction), $\supset$ (implication), and $\neg$ (negation), and quantifiers $\exists$ (existential quantifier) and $\forall$ (universal quantifier), a denumerable list of individual variables and a denumerable list of $m$-ary predicate variables for each $m<\omega$. The 0 -ary predicate variables are identified with propositional variables. Note that $\mathcal{L}$ contains neither constants nor function symbols, nor the equality $(=)$. For every formula $A$, we denote the set of all variables free in $A$ by $F V(A)$.

Roughly speaking, intermediate logics are logics located between intuitionistic and classical logics.

Definition 1.1. ( $c f$. Ono[11]) A set $\mathbf{L}$ of formulas of $\mathcal{L}$ is said to be an intermediate predicate logic, if $\mathbf{L}$ satisfies the following three conditions:

(Q1) $\mathbf{L}$ contains all formulas provable in intuitionistic predicate logic $\mathbf{H}_{*}$;

(Q2) every formula in $\mathbf{L}$ is provable in classical predicate logic $\mathbf{C}_{*}$;

(Q3) $\mathbf{L}$ is closed under the rule of modus ponens (from $A$ and $A \supset B$, infer $B$ ), the rule of generalization (from $A$, infer $\forall x A$ ), and uniform substitution $^{3}$ for predicate variables.

A set $\mathbf{L}$ of formulas of $\mathcal{L}$ is said to be a super-intuitionistic predicate logic, if $\mathbf{L}$ satisfies (Q1) and (Q3).

We sometimes use the phrase " $A$ is provable in $\mathbf{L}$ " (in symbol: $\mathbf{L} \vdash A$ ) when $A \in \mathbf{L}$. We identify a logic with the sets of formulas provable in it. For every formula $A$, the smallest super-intuitionistic predicate logic containing $A$ is denoted by $\mathbf{H}_{*}+A$. Hereafter, we sometimes omit "intermediate predicate" and "super-intuitionistic predicate" for the sake of simplicity.

${ }^{3} C f$. the operator $\breve{S}$ in Church [1]. 
Definition 1.2. (cf. Kleene [6]) Formulas $A$ and $B$ are congruent, if $A$ is obtained from $B$ by alphabetic change of bound variables which does not turn any free occurrences of newly bound variables ( $c f$. Kleene [5, p.153]). A logic $\mathbf{L}$ is said to have the existence property (EP), if for every $\exists x A(x)$, $\mathbf{L} \vdash \exists x A(x)$ implies that there exist a formula $\tilde{A}(x)$ which is congruent to $A(x)$ and an individual variable $v$ such that $v$ is free for $x$ in $\tilde{A}(x)$ and $\mathbf{L} \vdash \tilde{A}(v)$.

Note that congruent formulas are equivalent to each other in intuitionistic logic. In the rest of this paper, we identify congruent formulas without mentioning it, whenever this is not likely to cause confusion ( $c f$. GabbayShehtman-Skvortsov [3, Ch.2]). Then, the definition of EP can be written simply as that in Introduction.

In [16], we used the following extremely strong EP in super-intuitionistic predicate logic: for every $\exists x A(x), \mathbf{L} \vdash \exists x A(x)$ implies that there exists a fresh individual variable $v$ such that $\mathbf{L} \vdash A(v)$. Clearly, this property is so extreme that no intermediate predicate logic has this property. However, if we restrict $\exists x A(x)$ to a sentence, we obtain one weak variant of EP:

Definition 1.3. A logic $\mathbf{L}$ is said to have the sentential existence property (sEP), if for every sentence $\exists x A(x)$ provable in $\mathbf{L}$, there exists a fresh individual variable $v$ such that $A(v)$ is provable in $\mathbf{L}$.

Next, we introduce the weak existence property (wEP). This property was originally considered in Prawitz [12] in the language with constant symbols but without function symbols ${ }^{4}$ to establish EP of intuitionistic predicate logic. Komori [7] and Minari [8] also used this property to establish EP of some intermediate predicate logics. Note that we (and Komori and Minari) are working with the pure language $\mathcal{L}$ having neither constant symbols nor function symbols. Then, for each formula $A$, the set $F V(A)$ is essentially the set of all terms constructed with the vocabulary of $A$. Thus, we have the following definition adapted to the language $\mathcal{L}$.

Definition 1.4. A logic $\mathbf{L}$ is said to have the weak existence property $(\mathrm{wEP}$ ), if for every formula $\exists x A(x)$ provable in $\mathbf{L}$ and every finite nonempty set $\left\{v_{1}, \ldots, v_{n}\right\}$ of individual variables such that $F V(\exists x A(x)) \subseteq$ $\left\{v_{1}, \ldots, v_{n}\right\}$, the disjunction $A\left(v_{1}\right) \vee \cdots \vee A\left(v_{n}\right)$ is provable in $\mathbf{L}$.

\footnotetext{
${ }^{4}$ And it was extended to that with the language with function symbols in Doorman [2].
} 
Then, we have the following Proposition.

Proposition 1.5. (1) EP implies wEP.

(2) wEP implies sEP.

Proof: (1): Suppose that a logic $\mathbf{L}$ has EP, and $\mathbf{L} \vdash \exists x A(x)$. Let $\left\{v_{1}, \ldots, v_{n}\right\}$ be an arbitrary finite set of variables such that $F V(\exists x A(x)) \subseteq$ $\left\{v_{1}, \ldots, v_{n}\right\}$. Since $\mathbf{L}$ has EP, there exists a variable $w$ such that $\mathbf{L} \vdash A(w)$. If $w$ is a variable free in $\exists x A(x)$, it is clear that $A\left(v_{1}\right) \vee \cdots \vee A\left(v_{n}\right)$ is provable in $\mathbf{L}$. If $w$ is a fresh variable, then, by the rule of generalization, we have $\mathbf{L} \vdash \forall w A(w)$. Therefore, we have $\mathbf{L} \vdash A\left(v_{1}\right)$, i.e., $\mathbf{L} \vdash A\left(v_{1}\right) \vee \cdots \vee A\left(v_{n}\right)$.

(2): Suppose that a logic $\mathbf{L}$ has $w E P$, and that $\exists x A(x)$ is a sentence provable in $\mathbf{L}$. Let $w$ be a fresh variable. Since $F V(\exists x A(x))$ is empty, we have $F V(\exists x A(x)) \subseteq\{w\}$. Then, $A(w)$ is provable in $\mathbf{L}$.

Now, we introduce the $Z$-normality, which is a weak variant of DP in intermediate predicate logics.

Definition 1.6. ([16]) Consider the following inference rule $(Z \mathrm{R})$ :

$$
\frac{A \vee(p(x) \supset p(y))}{A}(Z \mathrm{R})
$$

- subject to: $p$ is a unary predicate variable, and $x$ and $y$ are mutually distinct individual variables, and $p, x$, and $y$ do not occur in $A$.

A logic $\mathbf{L}$ is said to be $Z$-normal, if $\mathbf{L}$ is closed under $(Z \mathrm{R})$.

The rule $(Z R)$ is admissible both in $\mathbf{C}_{*}$ and in $\mathbf{H}_{*}$. Since $p(x) \supset p(y)$ is not provable in $\mathbf{C}_{*}$, we have that DP implies $Z$-normality in intermediate predicate logics. Since $\mathbf{C}_{*}$ does not have DP, the converse does not hold, i.e., $Z$-normality does not imply $\mathrm{DP}$. The $Z$-normality is a natural property for reasonable logics ( $c f$. Proposition 2.4), and plays an important role in the consideration of the relation between EP and DP. Namely:

Theorem 1.7 ([16]). If a Z-normal logic has EP, then it has DP.

Then, a logic $\mathbf{L}$ has EP and is $Z$-normal if and only if $\mathbf{L}$ has both EP and DP, and if and only if $\mathbf{L}$ has both wEP and DP. In symbol:

$$
\mathrm{EP}+Z \text {-normal } \leftrightarrow \mathrm{EP}+\mathrm{DP} \leftrightarrow \mathrm{wEP}+\mathrm{DP}
$$

Thus, we illustrate the situation in Figure 1. More precisely: 


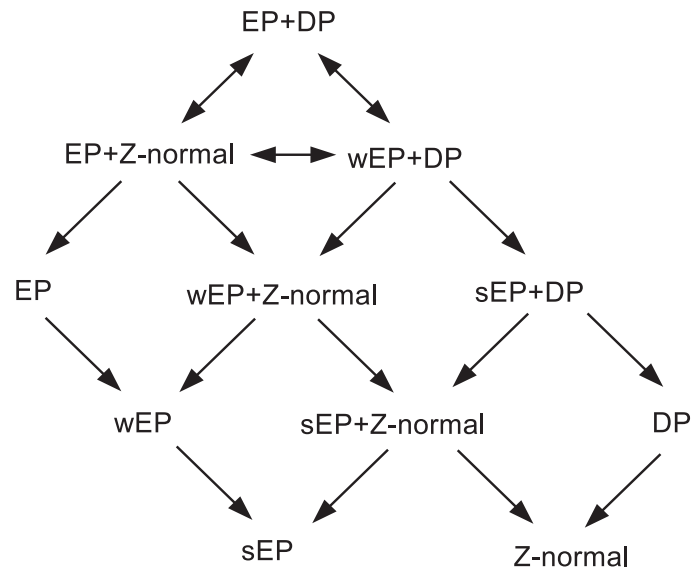

Fig. 1.

THEOREM 1.8. The Figure 1 comprehensively describes the situation. There are no additional $\rightarrow$ 's of implication in Figure 1.

The aim of this paper is to show Theorem 1.8. It suffices to show that the following four kinds of logics exit:

- $\mathbf{L}_{1}$ having EP, but not being $Z$-normal,

- $\mathbf{L}_{2}$ having wEP and being $Z$-normal, but lacking EP and DP,

- $\mathbf{L}_{3}$ having sEP and DP, but lacking wEP,

- $\mathbf{L}_{4}$ having DP, but lacking sEP.

In [16], we constructed logics ${ }^{5}$ having EP but lacking DP. These logics are not $Z$-normal by Theorem 1.7. Hence one of these logics can be used as $\mathbf{L}_{1}$. Nakamura [9] and Minari [8] proved that DP does not imply EP by constructing logics having DP but lacking EP. In fact, they showed that there are logics which have DP but lack sEP. Hence, their logics serve as $\mathbf{L}_{4}$. Thus, it remains to find $\mathbf{L}_{2}$ and $\mathbf{L}_{3}$. In $\S 3$, we will give two concrete finitely axiomatizable logics $\mathbf{L}_{2}$ and $\mathbf{L}_{3}$ and show that they fulfill the above requirements. Our idea of the proof is essentially based on Nakamura [9]

\footnotetext{
${ }^{5}$ Some of them are proved to be finitely axiomatizable.
} 
and Minari [8], with some modifications using the Kripke-sheaf semantics. (cf. also [13].)

\section{Kripke sheaf semantics}

First, we give a short explanation of the Kripke sheaf semantics for intermediate predicate logics. Then, we give semantical constructions and a Lemma for proving Theorem 1.8.

\subsection{Kripke sheaves}

For every non-empty set $U$, the language obtained from $\mathcal{L}$ by adding the name $\bar{u}$ for each $u \in U$ is denoted by $\mathcal{L}[U]$. In what follows, we will sometimes use the same letter $u$ for the name $\bar{u}$ of $u$. Let $U$ and $V$ be non-empty sets, $\varphi: U \rightarrow V$ a mapping. For each formula $A$ of $\mathcal{L}[U]$, by $A^{\varphi}$ we denote a formula obtained from $A$ by replacing all occurrences of $\bar{u}$ by the name $\overline{\varphi(u)}$ of $\varphi(u)$ for each $u \in U$. That is, $\varphi$ naturaly induces a translation of $\mathcal{L}[U]$ into $\mathcal{L}[V]$. This notation is also used for the substitution of names of individuals in a set $V$ for free occurrences of individual variables. Namely, $A^{\varphi}$ with a mapping $\varphi$ of a set $\left\{x_{i_{1}}, x_{i_{2}}, \ldots\right\}$ of individual variables to $U$ denotes the formula of $\mathcal{L}[U]$ obtained from $A$ by replacing all free occurrences of $x_{i_{j}}$ by the name $\overline{\varphi\left(x_{i_{j}}\right)}$ of $\varphi\left(x_{i_{j}}\right) \in U$ for each individual variable $x_{i_{j}}$ in $\left\{x_{i_{1}}, x_{i_{2}}, \ldots\right\}$.

Definition 2.1. A partially ordered set $\mathbf{M}=(M, \leq)$ with the least element $0_{\mathbf{M}}$ is said to be a Kripke base. We can regard a Kripke base $\mathbf{M}$ as a category in the usual way. Let $\mathcal{S}$ ets be the category of all sets. A covariant functor $D$ from a Kripke base $\mathbf{M}$ to $\mathcal{S}$ ets is called a domain-sheaf over $\mathbf{M}$, if $D(a)$ is non-empty for every $a \in M$. That is,

(DS1) $D(a) \neq \varnothing$ for every $a \in M$,

(DS2) for every $a, b \in M$ with $a \leq b$, a mapping $D_{a b}: D(a) \rightarrow D(b)$ is associated,

(DS3) $D_{a a}$ is the identity mapping $i d_{D(a)}$ of $D(a)$ for every $a \in M$, (DS4) $D_{a c}=D_{b c} \circ D_{a b}$ for every $a, b, c \in M$ with $a \leq b \leq c$.

A pair $\mathcal{K}=\langle\mathbf{M}, D\rangle$ of a Kripke base $\mathbf{M}$ and a domain-sheaf $D$ over $\mathbf{M}$ is called a Kripke sheaf. If every $D_{a b}(a \leq b)$ is the set-inclusion, $\langle\mathbf{M}, D\rangle$ is said to be a Kripke frame. 
Intuitively, each $D(a)$ is the individual domain of the world $a \in M$. If $a \leq b$, then each $d \in D(a)$ has its inheritor in $D(b)$, i.e., $D_{a b}(d) \in D(b)$. For each $a \in M$ and each $b \in M$ with $a \leq b$, every sentence $A$ of $\mathcal{L}[D(a)]$ is translated into the sentence $A^{D_{a b}}$ of $\mathcal{L}[D(b)]$, which is called the inheritor of $A$ at $b$.

A binary relation $\models$ between each $a \in M$ and each atomic sentence of $\mathcal{L}[D(a)]$ is said to be a valuation on $\mathcal{K}=\langle\mathbf{M}, D\rangle$, if for every $a, b \in M$ and every atomic sentence $A$ of $\mathcal{L}[D(a)], a \models A$ and $a \leq b$ imply $b \models A^{D_{a b}}$. We inductively extend $\models$ to a relation between each $a \in M$ and each sentence of $\mathcal{L}[D(a)]$ in the following way:

- $a \models A \wedge B$ if and only if $a \models A$ and $a \models B$,

- $a \models A \vee B$ if and only if $a \models A$ or $a \models B$,

- $a \models A \supset B$ if and only if for every $b \in M$ with $a \leq b$, either $b \not \models A^{D_{a b}}$ or $b \models B^{D_{a b}}$,

- $a \models \neg A$ if and only if for every $b \in M$ with $a \leq b, b \not \models A^{D_{a b}}$,

- $a \models \forall x A(x)$ if and only if for every $b \in M$ with $a \leq b$ and every $u \in D(b), b \models A^{D_{a b}}(\bar{u})$,

- $a \models \exists x A(x)$ if and only if there exists $u \in D(a)$ such that $a \models A(\bar{u})$.

A pair $(\mathcal{K}, \models)$ of a Kripke sheaf $\mathcal{K}$ and a valuation $\models$ on it is said to be a Kripke model. A formula $A$ of $\mathcal{L}$ is said to be true in a Kripke model $(\mathcal{K}, \models)$, if $0_{\mathrm{M}} \models \bar{A}$, where $\bar{A}$ is the universal closure of $A$. A formula of $\mathcal{L}$ is said to be valid in a Kripke sheaf $\mathcal{K}$, if it is true in $(\mathcal{K}, \models)$ for every valuation $\models$ on $\mathcal{K}$. The set of formulas of $\mathcal{L}$ that are valid in $\mathcal{K}$ is denoted by $L(\mathcal{K})$. The following two propositions are fundamental properties of the Kripke sheaf semantics ( $c f$. Suzuki $[13,14])$.

Proposition 2.2. For every $a, b \in M$, and every sentence $A$ of $\mathcal{L}[D(a)]$, if $a \models A$ and $a \leq b$, then $b \models A^{D_{a b}}$.

Proposition 2.3. For each Kripke sheaf $\mathcal{K}$, the set $L(\mathcal{K})$ is a superintuitionistic predicate logic.

A logic $\mathbf{L}$ is said to be characterized by a Kripke sheaf $\mathcal{K}$, if $\mathbf{L}=L(\mathcal{K})$. A logic $\mathbf{L}$ is said to be characterized by a class $\mathcal{C}$ of Kripke sheaves, if $\mathbf{L}=\bigcap\{L(\mathcal{K}) ; \mathcal{K} \in \mathcal{C}\}$. Let $\mathcal{F}$ be a class of Kripke bases. A predicate logic $\mathbf{L}$ is said to be characterized by $\mathcal{F}$, if $\mathbf{L}$ is characterized by the class $\{\langle\mathbf{M}, D\rangle ; \mathbf{M} \in \mathcal{F}$ and $D$ is a domain-sheaf over $\mathbf{M}\}$. 
In Introduction, we stated that $Z$-normality is a natural property for semantically reasonable logics. More precisely, we have the following Proposition. This Proposition suggests that most of logics manageable with Kripke sheaves are automatically $Z$-normal.

Proposition 2.4. (1) Suppose that a super-intuitionistic predicate logic $\mathbf{L}$ is $Z$-normal. Then, $\mathbf{L}$ is characterized by a class of Kripke sheaves if and only if there exists a class $\mathcal{C}$ of Kripke sheaves such that $\mathbf{L}$ is characterized by $\mathcal{C}$ and each member $\mathcal{K}=\langle\mathbf{M}, D\rangle$ of $\mathcal{C}$ has at least two elements in $D\left(0_{\mathbf{M}}\right)$.

(2) If a super-intuitionistic predicate logic $\mathbf{L}$ is characterized by a class of Kripke bases, then $\mathbf{L}$ is $Z$-normal.

\subsection{Semantical toolkit}

Here we introduce two constructions of Kripke sheaves from given Kripke sheaves. The ideas of these constructions can be found in Nakamura [9] and Minari [8] originally for Kripke frames.

Definition 2.5. (cf. Minari [8]) Let $\mathcal{K}=\langle\mathbf{M}, D\rangle$ be a Kripke sheaf, $V$ a non-empty set. Suppose we have a mapping $f: V \rightarrow D\left(0_{\mathbf{M}}\right)$ where $0_{\mathbf{M}}$ is the least element of $\mathbf{M}$. Take a fresh element 0 and define a Kripke base $0 \uparrow \mathbf{M}$ as the partially ordered set obtained from $\mathbf{M}$ by adding 0 as the new least element. Then, $0 \uparrow \mathbf{M}$ and $f$ naturally induce a Kripke sheaf by associating the domain-sheaf $D_{f}^{\triangleright}$ :

$$
D_{f}^{\triangleright}(a)= \begin{cases}V & \text { if } a=0, \\ D(a) & \text { if } a \in \mathbf{M} ;\end{cases}
$$

for every $a \leq b$,

$$
\left(D_{f}^{\triangleright}\right)_{a b}= \begin{cases}i d_{V} & \text { if } a=b=0, \\ D_{0_{\mathbf{M}} b} \circ f & \text { if } a=0 \text { and } b \in \mathbf{M}, \\ D_{a b} & \text { if } a, b \in \mathbf{M} .\end{cases}
$$

This Kripke sheaf is denoted by $V \triangleright_{f} \mathcal{K}$, (or simply by $V \triangleright \mathcal{K}$ ), which we call the pointed extension of $\mathcal{K}$. We omit the subscript $\bullet_{f}$, whenever this is not likely to cause confusion.

Definition 2.6. (cf. Minari [8] and Nakamura [9]) Let $\mathcal{K}_{1}=\left\langle\mathbf{M}_{1}, D_{1}\right\rangle$ and $\mathcal{K}_{2}=\left\langle\mathbf{M}_{2}, D_{2}\right\rangle$ be Kripke sheaves with the least elements $0_{1}$ and $0_{2}$, 
respectively, $V$ a non-empty set. Suppose we have two mappings $f_{1}: V \rightarrow$ $D_{1}\left(0_{1}\right)$ and $f_{2}: V \rightarrow D_{2}\left(0_{2}\right)$. Take a fresh element 0 and define a Kripke base $0 \uparrow\left(\mathbf{M}_{1} \oplus \mathbf{M}_{2}\right)$ as the partially ordered set obtained from the disjoint union of $\mathbf{M}_{1}$ and $\mathbf{M}_{2}$ by adding 0 as the new least element. Then, $0 \uparrow$ $\left(\mathbf{M}_{1} \oplus \mathbf{M}_{2}\right), f_{1}$, and $f_{2}$ naturally induce a Kripke sheaf $V \uparrow_{f_{1}, f_{2}}\left(\mathcal{K}_{1} \oplus \mathcal{K}_{2}\right)$ (or simply, $V \uparrow\left(\mathcal{K}_{1} \oplus \mathcal{K}_{2}\right)$ ) by associating the domain-sheaf $D_{f_{1}, f_{2}}^{\uparrow}$ :

$$
D_{f_{1}, f_{2}}^{\uparrow}(a)= \begin{cases}V & \text { if } a=0 \\ D(a) & \text { if } a \in \mathbf{M}_{1} \oplus \mathbf{M}_{2}\end{cases}
$$

for every $a \leq b$,

$$
\left(D_{f_{1}, f_{2}}^{\uparrow}\right)_{a b}= \begin{cases}i d_{V} & \text { if } a=b=0 \\ \left(D_{i}\right)_{0_{i} b} \circ f_{i} & \text { if } a=0 \text { and } b \in \mathbf{M}_{i}(i=1,2) \\ \left(D_{i}\right)_{a b} & \text { if } a \in \mathbf{M}_{i}(i=1,2)\end{cases}
$$

The Kripke sheaf $V \uparrow_{f_{1}, f_{2}}\left(\mathcal{K}_{1} \oplus \mathcal{K}_{2}\right)$ is called the pointed join of $\mathcal{K}_{1}$ and $\mathcal{K}_{2}$ (with $V, f_{1}$, and $f_{2}$ ). We omit the subscript $\bullet_{f_{1}, f_{2}}$, whenever this is not likely to cause confusion.

Definition 2.7. For every Kripke model $(\mathcal{K}, \models)$, there exists at least one valuation $\models^{\prime}$ on $V \triangleright \mathcal{K}$ such that $\models$ and $\models^{\prime}$ concide on $\mathcal{K}$. Such a valuation is said to be an extension of $\models$. Similarly, we define an extension $\models$ of $\models_{1}$ and $\models_{2}$ on $V \uparrow\left(\mathcal{K}_{1} \oplus \mathcal{K}_{2}\right)$ for each pair $\left(\mathcal{K}_{1}, \models_{1}\right)$ and $\left(\mathcal{K}_{2}, \models_{2}\right)$ of Kripke models.

Next, we prepare a Lemma, which we will use in $\S 3$.

Let $p$ be an $n$-ary predicate variable, $q$ a propositional variable. We denote the propositional formula $q \supset q$ by $\top$. Since $\top$ has no free variable, we can uniformly substitute $\top$ for $p\left(v_{1}, \ldots, v_{n}\right)$ in any formula $A$; the resulting formula is denoted by $A^{\top}$ (i.e., $\check{\mathrm{S}}_{\top}^{p\left(v_{1}, \ldots, v_{n}\right)} A \mid$ ).

Lemma 2.8. Let $(\mathcal{K}, \models)=(\langle\mathbf{M}, D\rangle, \models)$ be a Kripke model, $p$ an n-ary predicate variable. Suppose that $0_{\mathbf{M}} \models \forall x_{1} \ldots \forall x_{n} p\left(x_{1}, \ldots, x_{n}\right)$. Then, for every formula $A$ of $\mathcal{L}$, every $m \in \mathbf{M}$, every mapping $\varphi$ of $F V(A)$ to $D(m)$, it holds that $m \models A^{\varphi}$ if and only if $m \models\left(A^{\top}\right)^{\varphi}$.

Proof: We show this Lemma by induction on $A$. If $A$ is atomic, then the statement holds by the assumption. Suppose that $A$ is of the form $\neg B$. If $m \not \models \neg B^{\varphi}$, then there exists $m^{\prime} \in \mathbf{M}$ such that $m \leq m^{\prime}$ and $m^{\prime} \models$ $\left(B^{\varphi}\right)^{D_{m m^{\prime}}}$. Note that $\left(B^{\varphi}\right)^{D_{m m^{\prime}}}$ is $B^{\left(D_{m m^{\prime}} \circ \varphi\right)}$. By induction hypothesis, 
we have $m^{\prime}=\left(B^{\top}\right)^{\left(D_{m m^{\prime}} \circ \varphi\right)}$, that is, $m^{\prime} \models\left(\left(B^{\top}\right)^{\varphi}\right)^{D_{m m^{\prime}}}$. Then, it holds that $m \not \forall \neg\left(B^{\top}\right)^{\varphi}$. This reasoning can be applied backward. Next, suppose that $A$ is of the form $\forall z B(z)$. If $m \not \forall(\forall z B(z))^{\varphi}$, then there are $m^{\prime} \in \mathbf{M}$

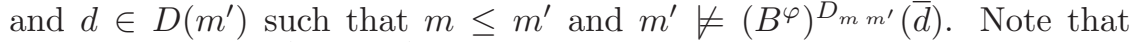
$\left(B^{\varphi}\right)^{D_{m m^{\prime}}}(\bar{d})$ is $B^{\left(D_{m m^{\prime}} \circ \varphi\right)}(\bar{d})$. Define a mapping $\psi$ of $F V(B(z))$ to $D\left(m^{\prime}\right)$ by:

$$
\psi(v)= \begin{cases}D_{m m^{\prime}} \circ \varphi(v) & \text { if } v \text { is not } z \\ d & \text { if } v \text { is } z\end{cases}
$$

Then, we have $m^{\prime} \not \forall B(z)^{\psi}$. By induction hypothesis, it holds that $m^{\prime} \not \models$ $\left(B(z)^{\top}\right)^{\psi}$. Namely, $m^{\prime} \not \models\left(\left(B^{\top}\right)^{\varphi}\right)^{D_{m m^{\prime}}}(\bar{d})$, and hence $m \not \forall\left(\forall z B(z)^{\top}\right)^{\varphi}$. This reasoning can be applied backward. Other cases can be treated similarly.

\section{Proof of the Theorem}

Let $p$ and $q$ be mutually distinct propositional variables, $r$ a binary predicate variable. Define two sentences $\operatorname{Lin}$, and $T_{1}$, and a formula $T(a, b, w)$ as follows:

$$
\begin{aligned}
\text { Lin } & : \quad(p \supset q) \vee(q \supset p) \\
T_{1} & : \quad \forall x \forall y \exists z(r(x, y) \supset r(x, z) \wedge r(y, z)) \\
T(a, b, w) & : \quad T_{1} \wedge r(a, b) \supset r(a, w) \wedge r(b, w) .
\end{aligned}
$$

Next, define two logics as follows:

$$
\begin{aligned}
& \mathbf{L}_{2}=\mathbf{H}_{*}+\text { Lin. } \\
& \mathbf{L}_{3}=\mathbf{H}_{*}+\exists w T(a, b, w) .
\end{aligned}
$$

Since Lin and $\exists w T(a . b . w)$ are provable in classical logic $\mathbf{C}_{*}$, both of $\mathbf{L}_{2}$ and $\mathbf{L}_{3}$ are intermediate logics.

Lemma 3.1. The logic $\mathbf{L}_{2}$ is a $Z$-normal intermediate logic having wEP, but lacking EP and DP.

Proof: Minari [8] proved that $\mathbf{L}_{2}$ has wEP and lacks EP and DP. We show that $\mathbf{L}_{2}$ is $Z$-normal ${ }^{6}$. Suppose that $A$ is not provable in $\mathbf{L}_{2}$. We will

\footnotetext{
${ }^{6}$ It is obvious by the definition that $\mathbf{L}_{2}$ lacks DP. By Proposition 1.7, the failure of EP follows from the $Z$-normality of $\mathbf{L}_{2}$.
} 
show that $\mathbf{L}_{2} \nvdash A \vee(p(x) \supset p(y))$, where the unary predicate variable $p$ and individual variables $x$ and $y$ do not occur in $A$. Without loss of generality, we may assume that $A$ contains no free variables other than $v_{1}, \ldots, v_{n}$, and we write $A$ as $A\left(v_{1}, \ldots, v_{n}\right)$. Then, by the strong completeness theorem of $\mathbf{H}_{*}$ with respect to the Kripke semantics, there is a Kripke model $(\mathcal{K}, \models)=(\langle\mathbf{M}, D\rangle, \models)$ such that:

(M1) $0_{\mathbf{M}} \models \overline{(X \supset Y) \vee(Y \supset X)}$, for all formulas $X$ and $Y$ of $\mathcal{L}$, (M2) there are $d_{1}, \ldots, d_{n} \in D\left(0_{\mathbf{M}}\right)$ such that $0_{\mathbf{M}} \not \models A\left(d_{1}, \ldots, d_{n}\right)$,

where $0_{\mathbf{M}}$ is the least element of $\mathbf{M}$. Take two fresh elements $e_{1}$ and $e_{2}$, and define a set $V=\left\{d_{1}, \ldots, d_{n}, e_{1}, e_{2}\right\}$ and a mapping $f: V \rightarrow D\left(0_{\mathbf{M}}\right)$ by

$$
f(d)= \begin{cases}d & \text { if } d=d_{i} \text { for some } i=1, \ldots, n \\ d_{1} & \text { if } d=e_{1} \text { or } d=e_{2} .\end{cases}
$$

Then, we have the pointed extension $V \triangleright_{f} \mathcal{K}$ with the new least element 0 . By extending $\models$, we have a valuation on $V \triangleright_{f} \mathcal{K}$, which we denote by the same symbol $\models$. Clearly, by (M2), we have $0 \not \models A\left(d_{1}, \ldots, d_{n}\right)$. Modifying $\models$, we define $\models{ }^{\prime}$ as follows:

For each $m \in 0 \uparrow \mathbf{M}$ and each atomic sentence $B$ of $\mathcal{L}\left[D^{\triangleright}(m)\right]$, $m \models{ }^{\prime} B$ if and only if

$$
\begin{cases}m \models B & \text { if } B \text { is not of the form } p(d), \\ m \neq 0 \text { or } d=e_{1} & \text { if } B \text { is of the form } p(d) .\end{cases}
$$

Then, it is easy to see that $0 \not \nvdash^{\prime} A\left(d_{1}, \ldots, d_{n}\right) \vee\left(p\left(e_{1}\right) \supset p\left(e_{2}\right)\right)$. It remains to show that

$(* 1) 0 \models^{\prime} \overline{(X \supset Y) \vee(Y \supset X)}$ for all formulas $X$ and $Y$ of $\mathcal{L}$.

By the definition of $\models^{\prime}$, we have $0_{\mathbf{M}} \models^{\prime} \forall x p(x)$. Then, by Lemma 2.8, we have that, for every sentence $W$ of $\mathcal{L}\left[D\left(0_{\mathbf{M}}\right)\right]$,

$$
0_{\mathbf{M}} \models^{\prime} W \text { if and only if } 0_{\mathbf{M}} \models^{\prime} W^{\top} \text {. }
$$

Since $W^{\top}$ contains no occurrence of $p$, two valuations, $\models^{\prime}$ and $\models$ on $\mathcal{K}$ coincide with each other for such sentences. Therefore,

$$
0_{\mathbf{M}} \models^{\prime} W^{\top} \text { if and only if } 0_{\mathbf{M}} \models W^{\top} \text {. }
$$


Recall that $\overline{(X \supset Y) \vee(Y \supset X)}^{\top}$ is also the universal closure of an instance of Lin. Taking this sentence as $W$ above, by (M1), we have:

(M3) $0_{\mathbf{M}} \models^{\prime} \overline{(X \supset Y) \vee(Y \supset X)}$ for all $X$ and $Y$ of $\mathcal{L}$.

Now, we show $(* 1)$. Suppose otherwise. Then, there exist $X$ and $Y$

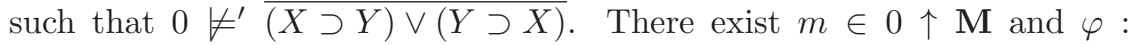
$F V(X) \cup F V(Y) \rightarrow D^{\triangleright}(m)$ such that $m \not \not^{\prime}\left(X^{\varphi} \supset Y^{\varphi}\right) \vee\left(Y^{\varphi} \supset X^{\varphi}\right)$. By (M3), this $m$ must be 0 . Hence, $0 \not \forall^{\prime} X^{\varphi} \supset Y^{\varphi}$ and $0 \not \nvdash^{\prime} Y^{\varphi} \supset X^{\varphi}$. Then, there are $k$ and $\ell$ in $0 \uparrow \mathbf{M}$ such that

(M4) $k \models^{\prime}\left(X^{\varphi}\right)^{D_{0 k}^{\triangleright}}, k \nvdash^{\prime}\left(Y^{\varphi}\right)^{D_{0 k}^{\triangleright}}, \ell \models^{\prime}\left(Y^{\varphi}\right)^{D_{0 \ell}^{\triangleright}}$, and $\ell \not \models^{\prime}\left(X^{\varphi}\right)^{D_{0}^{\triangleright} \ell}$.

Assume that $0_{\mathbf{M}} \leq k, \ell$. Then, we have:

$$
D_{0 k}^{\triangleright} \circ \varphi=\left(D_{0_{\mathrm{M}} k}^{\triangleright} \circ D_{00_{\mathrm{M}}}^{\triangleright}\right) \circ \varphi=D_{0_{\mathrm{M} k}}^{\triangleright} \circ\left(D_{00_{\mathrm{M}}}^{\triangleright} \circ \varphi\right) .
$$

Thus, $\left(X^{\varphi}\right)^{D_{0}^{\triangleright} k}$ and $\left(Y^{\varphi}\right)^{D_{0 k}^{\triangleright}}$ are $\left(X^{\left(D_{0}^{\triangleright} 0_{\mathbf{M}} \circ \varphi\right)}\right)^{D_{0_{\mathbf{M}} k}^{\triangleright}}$ and $\left(Y^{\left(D_{0}^{\triangleright} 0_{\mathbf{M}}\right.} \circ \varphi\right){ }^{D_{0_{\mathbf{M}} k}^{\triangleright}}$, respectively. By (M4), it holds that $0_{\mathbf{M}} \not \nvdash^{\prime} X^{\left(D_{0}^{\triangleright} 0_{\mathbf{M}} \circ \varphi\right)} \supset Y^{\left(D_{0}^{\triangleright} 0_{\mathbf{M}}\right.}{ }^{\circ \varphi)}$. Similarly, by replacing $k$ by $\ell$, we have: $0_{\mathbf{M}} \not \models^{\prime} Y^{\left(D_{0}^{\triangleright} 0_{\mathbf{M}} \circ \varphi\right)} \supset X^{\left(D_{0}^{\triangleright} 0_{\mathbf{M}} \circ \varphi\right)}$. That is, $0_{\mathbf{M}} \not \nvdash^{\prime}\{(X \supset Y) \vee(Y \supset X)\}^{\left(D_{0}^{\triangleright} 0_{\mathbf{M}}{ }^{\circ} \varphi\right)}$. This contradicts (M3). Therefore, $k=0$ or $\ell=0$. Without loss of generality, we may assume that $k=0$. Then, $0=k \leq \ell$. From (M4), we have that $0 \models^{\prime} X^{\varphi}$ and $\ell \not \not^{\prime}\left(X^{\varphi}\right)^{D_{0}^{\triangleright} \ell}$. This contradicts Proposition 2.2. Thus we have established $(* 1)$.

LEMma 3.2. The logic $\mathbf{L}_{3}$ is an intermediate logic having sEP and DP, but lacking $w E P$.

Proof: Since $T(a, b, a) \vee T(a, b, b)$ is not provable in $\mathbf{C}_{*}, \mathbf{L}_{3}$ fails to have wEP. It suffices to show that $\mathbf{L}_{3}$ has $\mathrm{sEP}$ and DP.

First, we show that $\mathbf{L}_{3}$ has sEP. Suppose that $\exists x A(x)$ is a sentence. Let $v$ be a fresh individual variable free for $x$ in $A(x)$. Suppose, moreover, that $\mathbf{L}_{3} \nvdash A(v)$. Then, by the strong completeness theorem of $\mathbf{H}_{*}$, there is a Kripke model $(\mathcal{K}, \models)=(\langle\mathbf{M}, D\rangle, \models)$ such that

(L1) $0_{\mathbf{M}} \models \bar{X}$ for every instance $X$ of $T$ in $\mathcal{L}$,

(L2) there exists $d \in D\left(0_{\mathbf{M}}\right)$ such that $0_{\mathbf{M}} \not \models A(d)$.

Put $V=\{d\}$, and make the pointed extension $V \triangleright \mathcal{K}$. By extending $\models$ to $V \triangleright \mathcal{K}$, we have $0 \not \models A(d)$. Thus, $0 \not \models \exists x A(x)$. It remains to show that

$(* 2) 0 \models \bar{X}$ for every instance $X$ of $T$ in $\mathcal{L}$. 
Suppose otherwise. Then, there exists a formula $R(a, b, v)$ of $\mathcal{L}$ such that $R(a, b, v)$ contains no free variables other than $a, b$, and $v^{7}$ and that the universal closure of

$$
\begin{aligned}
\exists w[\{\forall x \forall y \exists z(R(x, y, v) \supset R(x, z, v) \wedge R(y, z, v))\} & \wedge R(a, b, v) \\
& \supset R(a, w, v) \wedge R(b, w, v)]
\end{aligned}
$$

is not true in $V \triangleright \mathcal{K}$. By the reasoning similar to that in Lemma 3.1, we have $0 \forall \forall \exists w[\{\forall x \forall y \exists z(R(x, y, d) \supset R(x, z, d) \wedge R(y, z, d))\} \wedge R(d, d, d) \supset$ $R(d, w, d) \wedge R(d, w, d)]$, since $D^{\triangleright}(0)=\{d\}$. Then, $0 \not \models\{\forall x \forall y \exists z(R(x, y, d) \supset$ $R(x, z, d) \wedge R(y, z, d))\} \wedge R(d, d, d) \supset R(d, d, d) \wedge R(d, d, d)]$. This is a contradiction. Thus we have $(* 2)$ and, hence $\mathbf{L}_{3}$ has sEP.

Next, we show that $\mathbf{L}_{3}$ has DP. Suppose that $\mathbf{L}_{3} \nvdash A$ and $\mathbf{L}_{3} \nvdash B$. Without loss of generality, we may assume that $A$ and $B$ contain no free variables other than $v_{1}, \ldots, v_{n}$, and we may write $A$ and $B$ as $A\left(v_{1}, \ldots, v_{n}\right)$ and $B\left(v_{1}, \ldots, v_{n}\right)$, respectively. Then, by the strong completeness theorem of $\mathbf{H}_{*}$, there are two Kripke models $\left(\mathcal{K}_{1}, \models_{1}\right)=\left(\left\langle\mathbf{M}_{1}, D_{1}\right\rangle, \models_{1}\right)$ and $\left(\mathcal{K}_{2}, \models_{2}\right)=\left(\left\langle\mathbf{M}_{2}, D_{2}\right\rangle, \models_{2}\right)$ such that:

(L3) for $i=1,2$, it holds that $0_{i} \models \bar{X}$ for every instance $X$ of $T$ in $\mathcal{L}$,

(L4-1) there are $d_{1}, \ldots, d_{n} \in D_{1}\left(0_{1}\right)$ such that $0_{1} \not \neq_{1} A\left(d_{1}, \ldots, d_{n}\right)$,

(L4-2) there are $e_{1}, \ldots, e_{n} \in D_{2}\left(0_{2}\right)$ such that $0_{2} \not \nvdash_{2} B\left(e_{1}, \ldots, e_{n}\right)$,

where $0_{i}$ is the least element of $\mathbf{M}_{i}(i=1,2)$. Let $V$ be the cartesian product $D_{1}\left(0_{1}\right) \times D_{2}\left(0_{2}\right)$. Then, we have the pointed join $V \uparrow_{\pi_{1}, \pi_{2}}\left(\mathcal{K}_{1} \oplus \mathcal{K}_{2}\right)$ by making use of the canonical projections $\pi_{i}: D_{1}\left(0_{1}\right) \times D_{2}\left(0_{2}\right) \rightarrow D_{i}\left(0_{i}\right)$ $(i=1,2)$. Check that the inheritor of $A\left(\left(d_{1}, e_{1}\right), \ldots,\left(d_{n}, e_{n}\right)\right)$ at $0_{1}$ is $A\left(d_{1}, \ldots, d_{n}\right)$, and that the inheritor of $B\left(\left(d_{1}, e_{1}\right), \ldots,\left(d_{n}, e_{n}\right)\right)$ at $0_{2}$ is $B\left(e_{1}, \ldots, e_{n}\right)$. Extending $\models$ to the pointed join, by (L4-1) and (L4-2), we have

$$
0 \not \models A\left(\left(d_{1}, e_{1}\right), \ldots,\left(d_{n}, e_{n}\right)\right) \vee B\left(\left(d_{1}, e_{1}\right), \ldots,\left(d_{n}, e_{n}\right)\right) .
$$

It remains to show that

$(* 3) 0 \models \bar{X}$ for every instance $X$ of $T$ in $\mathcal{L}$.

\footnotetext{
${ }^{7}$ In general, the $v$ may be a finite (possibly empty) sequence of individual variables.
} 
Suppose otherwise. With the same discussion as above, there exist a formula $R(a, b, v)$ of $\mathcal{L}$ and elements $c_{1}, c_{2}, c_{3} \in V$ such that $R(a, b, v)$ contains no free variables other than $a, b$, and $v$, and that

(L5) $0 \not \forall \exists w\left[\left\{\forall x \forall y \exists z\left(R\left(x, y, c_{3}\right) \supset R\left(x, z, c_{3}\right) \wedge R\left(y, z, c_{3}\right)\right)\right\} \wedge R\left(c_{1}, c_{2}, c_{3}\right)\right.$ $\left.\supset R\left(c_{1}, w, c_{3}\right) \wedge R\left(c_{2}, w, c_{3}\right)\right]$.

By (L3), there are $d_{1} \in D_{1}\left(0_{1}\right)$ and $d_{2} \in D_{2}\left(0_{2}\right)$ such that $0_{i} \models\left\{\forall x \forall y \exists z\left(R\left(x, y, c_{3}^{i}\right) \supset R\left(x, z, c_{3}^{i}\right) \wedge R\left(y, z, c_{3}^{i}\right)\right)\right\} \wedge R\left(c_{1}^{i}, c_{2}^{i}, c_{3}^{i}\right) \supset$ $R\left(c_{1}^{i}, d_{i}, c_{3}^{i}\right) \wedge R\left(c_{2}^{i}, d_{i}, c_{3}^{i}\right)$, where $c_{j}^{i}=\pi_{i}\left(c_{j}\right)$ for $i=1,2$ and $j=1,2,3$. Put $d=\left(d_{1}, d_{2}\right) \in V$. Then, by (L5), we have $0 \models\left\{\forall x \forall y \exists z\left(R\left(x, y, c_{3}\right) \supset\right.\right.$ $\left.\left.R\left(x, z, c_{3}\right) \wedge R\left(y, z, c_{3}\right)\right)\right\} \wedge R\left(c_{1}, c_{2}, c_{3}\right)$ and $0 \not \forall R\left(c_{1}, d, c_{3}\right) \wedge R\left(c_{2}, d, c_{3}\right)$. The former implies that there exists an element $e \in V$ such that $0 \models$ $R\left(c_{1}, e, c_{3}\right) \wedge R\left(c_{2}, e, c_{3}\right)$. Therefore, we have $0 \models \exists w\left[\left\{\forall x \forall y \exists z\left(R\left(x, y, c_{3}\right) \supset\right.\right.\right.$ $\left.\left.\left.R\left(x, z, c_{3}\right) \wedge R\left(y, z, c_{3}\right)\right)\right\} \wedge R\left(c_{1}, c_{2}, c_{3}\right) \supset R\left(c_{1}, w, c_{3}\right) \wedge R\left(c_{2}, w, c_{3}\right)\right]$. This contradicts (L5). Thus, we have $(* 3)$.

\section{Concluding remarks}

In this paper, we have determined the relationships among EP, DP, wEP, sEP, and $Z$-normality in the setting of intermediate predicate logics.

Here we make some remarks for further research topics.

1. We are working with the pure language $\mathcal{L}$. In [2], Doorman considered a property related to $\mathrm{wEP}$ in languages with function symbols. It must be interesting to consider counterparts of sEP and $Z$-normality in such languages. At present, we have only some partial results.

2. We have considered sEP as a weak variant of EP. It suggests that we can introduce the sentential DP (sDP): if $\mathbf{L} \vdash A \vee B$ and $A$ and $B$ are sentences, then either $\mathbf{L} \vdash A$ or $\mathbf{L} \vdash B$. We can show that $Z$-normality and sDP are independent in intermediate predicate logics; sDP $\nrightarrow Z$-normality, and $Z$ normality $\nrightarrow \mathrm{sDP}$. But, with the constant domain axiom CD: $\forall x(p(x) \vee q) \supset$ $\forall x p(x) \vee q$, we can show that sDP implies $Z$-normality. The situation including sDP and/or CD is not completely clear at present.

3. Let us say that a formula is axiomatically true in a Kripke model, if its instances are all true in the model. The key concept of the proof of Theorem 1.8 is the preservation of axiomatic truth of Lin and $T$ under pointed 
extensions and pointed joins. (See also Minari [8] and Nakamura [9].) There are several such formulas. For example,

$$
\begin{aligned}
M P & : \quad \forall x(p(x) \vee \neg p(x)) \wedge \neg \neg \exists x p(x) \supset \exists x p(x), \\
L P O & : \quad \forall x(p(x) \vee \neg p(x)) \supset \exists x p(x) \vee \neg \exists x p(x),
\end{aligned}
$$

where $p$ is a unary predicate variable. The former corresponds to Markov's principle in a logical axiomatic schema, and the latter to the limited principle of omniscience. These formulas (axiom schemata) have been discussed in metamathematical investigations of constructive systems ( $c f$. e.g. Ishihara-Nemoto [4] and Troelstra-van Dalen [17]). Usually, EP and DP are regarded as the distinguishing features of constructivity of $\mathbf{H}_{*}$. Both $M P$ and $L P O$ enlarge the concept of constructivity, particularly the concepts of $\checkmark$ and $\exists$ of intuitionistic logic. However, they still have EP and DP. Note that Lin and $\exists w T(a, b, w)$ do the same. This observation seems to suggest a new insight into both fields: of intermediate logics and of metamathematics of constructive systems, which would enhance our understanding of $\exists, \vee$, and the concept of constructivity.

\section{References}

[1] A. Church, Introduction to Mathematical Logic I, Princeton University Press, Princeton (1956).

[2] L. M. Doorman, A note on the existence property for intuitionistic logic with function symbols, Zeitschrift für Mathematische Logik und Grundlagen der Mathematik 36 (1990), pp. 17-21.

[3] D. M. Gabbay, V. B. Shehtman and D. P. Skvortsov, Quantification in nonclassical logic. Vol. 1, Studies in Logic and the Foundations of Mathematics, 153. Elsevier, Amsterdam (2009).

[4] H. Ishihara and T. Nemoto, A note on the independence of premiss rule, Mathematical Logic Quarterly, to appear.

[5] S. C. Kleene, Introduction to metamathematics, D. Van Nostrand Co., Inc., New York 1952.

[6] S. C. Kleene, Disjunction and existence under implication in elementary intuitionistic formalisms, Journal of Symbolic Logic 27 (1962), pp. 1118. (An addendum, the same journal 28 (1963), pp. 154-156.)

[7] Y. Komori, Some results on the super-intuitionistic predicate logics, Reports on Mathematical Logic 15 (1983), pp. 13-31. 
[8] P. Minari, Disjunction and existence properties in intermediate predicate logics, Atti del Congresso Logica e Filosofia della Scienza, oggi. San Gimignano, 7-11 dicembre 1983. Vol. I - Logica. (1986), CLUEB, Bologna (Italy).

[9] T. Nakamura, Disjunction property for some intermediate predicate logics, Reports on Mathematical Logic 15 (1983), pp. 33-39.

[10] H. Ono, A study of intermediate predicate logics, Publications of the Research Institute for Mathematical Sciences 8 (1972/73), pp. 61-649.

[11] H. Ono, Some problems in intermediate predicate logics, Reports on Mathematical Logic 21 (1987), pp. 55-67. (Supplement 22 (1988), pp. 117-118.)

[12] D. Prawitz, Natural deduction. A proof-theoretical study, Acta Universitatis Stockholmiensis. Stockholm Studies in Philosophy, No. 3 Almqvist \& Wiksell, Stockholm 1965. (Reprint: Dover Publications, 2006)

[13] N.-Y. Suzuki, A remark on the delta operation and the Kripke sheaf semantics in super-intuitionistic predicate logics, Bulletin of the Section of Logic, University of Łódź, 25 (1996), pp. 21-28.

[14] N.-Y. Suzuki, Algebraic Kripke sheaf semantics for non-classical predicate logics Studia Logica 63 (1999), pp. 387-416.

[15] N.-Y. Suzuki, Halldén-completeness in super-intuitionistic predicate logics, Studia Logica 73 (2003), pp. 113-130

[16] N.-Y. Suzuki, A negative solution to Ono's problem P52: Existence and disjunction properties in intermediate predicate Logics, to appear.

[17] A. S. Troelstra and D. van Dalen, Constructivism in mathematics, Vol. I. An introduction, Studies in Logic and the Foundations of Mathematics, 121. North-Holland, Amsterdam, 1988.

[18] T. Umezawa, "Chūkan jutsugo ronri" (Intermediate predicate logics) (in Japanese), Gekkan Masematikusu, Vol. 1, No. 2 (1980), pp. 162-168. Kaiyo Shuppan Co., Ltd.

Department of Mathematics

Faculty of Science

Shizuoka University

Ohya 836, Suruga-Ku Shizuoka 422-8529, Japan

e-mail: suzuki.nobuyuki@shizuoka.ac.jp 